\title{
Concentrator Optical Characterization Using Computer Mathematical Modelling and Point Source Testing
}

\section{E.W. Dennison \\ S.L. John \\ G.F. Trentelman}

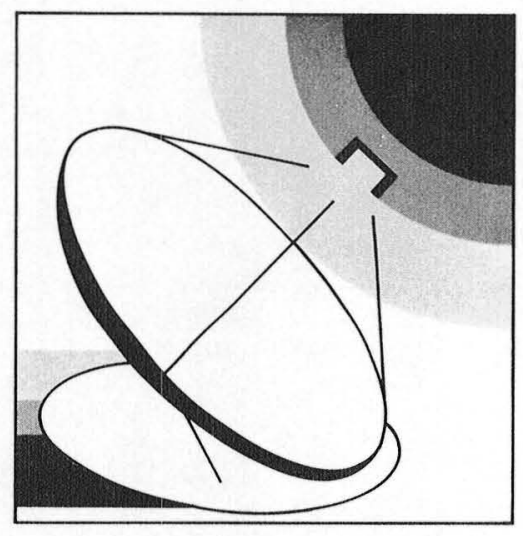

\section{LIBRARY COPY}

\section{APR $3 \quad 105$}

LANGLEY RESEARCH CENTER

LIBRARY, NASA

HAMPTON, VIRGINIA

September 15, 1984

Prepared for

U.S. Department of Energy

Through an Agreement with

National Aeronautics and Space Administration

by

Jet Propulsion Laboratory

California Institute of Technology

Pasadena, California

JPL Publication 84-75 



\title{
Concentrator Optical Characterization Using Computer Mathematical Modelling and Point Source Testing
}

\author{
E.W. Dennison \\ S.L. John \\ G.F. Trentelman
}

Prepared for

U.S. Department of Energy

Through an Agreement with

National Aeronautics and Space Administration

by

Jet Propulsion Laboratory

California Institute of Technology

Pasadena, California

JPL Publication 84-75 
Prepared by the Jet Propulsion Laboratory, California Institute of Technology, for the U.S. Department of Energy through an agreement with the National Aeronautics and Space Administration.

The JPL Solar Thermal Power Systems Project is sponsored by the U.S. Department of Energy and is part of the Solar Thermal Program to develop lowcost solar thermal and electric power plants.

This report was prepared as an account of work sponsored by an agency of the United States Government. Neither the United States Government nor any agency thereof, nor any of their employees, makes any warranty, express or implied, or assumes any legal liability or responsibility for the accuracy, completeness, or usefulness of any information, apparatus, product, or process disclosed, or represents that its use would not infringe privately owned rights.

Reference herein to any specific commercial product, process, or service by trade name, trademark, manuf acturer, or otherwise, does not necessarily constitute or imply its endorsement, recommendation, or favoring by the United States Government or any agency thereof. The views and opinions of authors expressed herein do not necessarily state or reflect those of the United States Government or any agency thereof. 
The optical characteristics of a paraboloidal solar concentrator are analyzed using the intercept factor curve (a format for image data) to describe the results of a mathematical model and to represent reduced data from experimental testing. This procedure makes it possible not only to test an assembled concentrator, but also to evaluate single optical panels or to conduct non-solar tests of an assembled concentrator.

The use of three-dimensional ray tracing computer programs to calculate the mathematical model is described. These ray tracing programs can include any type of optical configuration from simple paraboloids to arrays of spherical facets and can be adapted to microcomputers or larger computers, which can graphically display real-time comparison of calculated and measured data.

The technique described herein has demonstrated that

(1) It is possible to create a model for predicting concentrator optical performance from data obtained at various points of the experimental testing process, and that

(2) The intercept factor curve is a powerful format for optical performance representation -- whether it is derived from the mathematical model or from experimental data. 
The mathematical modelling described in this report was developed by coauthors George F. Trentelman and Susan L. John and was funded partly through a U.S. Department of Energy (DOE) summer research fellowship program and support from the Northern Michigan University, Marquette, Michigan. George (Fred) Trentelman is a member of the Physics Department at the University; Susan John is currently with GTE-Sylvania in Mountain View, California.

The work described herein was conducted by the Jet Propulsion Laboratory, California Institute of Technology, for the U.S. Department of Energy through an agreement with the National Aeronautics and space Administration (NASA Task RE-152, Amendment 327; DOE/ALO/NASA Interagency Agreement No. DE-AM04-80AL13137). 


\section{NOMENCLATURE}

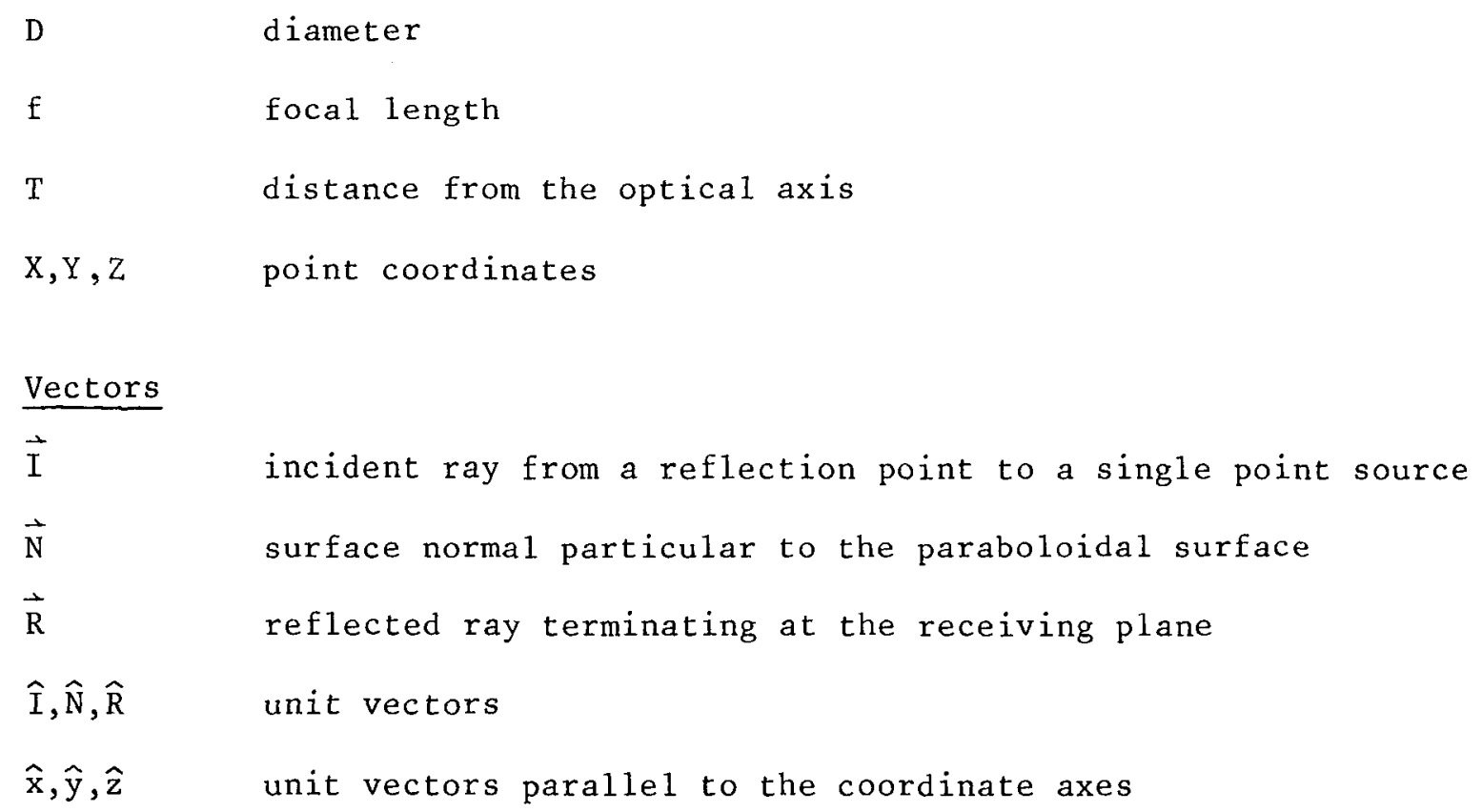

\section{Subscripts}

$\mathrm{m}$

$\mathrm{p}$

$\mathbf{S}$

$\mathrm{x}, \mathrm{y}, \mathrm{z}$ mirror coordinates relative to the vertex

coordinate of the reflected ray

coordinate of the point source relative to the vertex

components of a unit vector (direction cosine) 


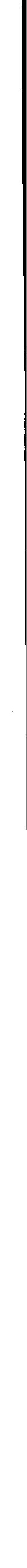


CONTENTS

I. INTRODUCTION . . . . . . . . . . . . . . . . . 1-1

II. COMPUTER MODEL . . . . . . . . . . . . . . . . . . 2-1

III. ABERRATIONAL EFFECTS AND IMPERFECT CONCENTRATORS . . . . . . . . 3-1

IV. RESULTS AND DISCUSSION . . . . . . . . . . . . . . . 4-1

A. FULL MIRROR COMPARISONS. . . . . . . . . . . . 4-1

B. POINT SOURCE TESTING OF INCOMPLETE MIRRORS . . . . . . 4-3

C. INTERCEPT FACTOR COMPARISONS WITH ONE PARAMETER. . . . . . 4-5

D. SOLAR EXTRAPOLATION. . . . . . . . . . . . . 4-6

v. Conclustons ........................ . . . . . 1

REFERENCES . . . . . . . . . . . . . . . . . . . . . R-1

APPENDIX: DATA TABLES FOR FIGURES IN TEXT. . . . . . . . . . . . A-1

\section{Figures}

2-1. Vector Analysis for Three-Dimensional Ray Tracing. . . . 2-2

2-2. Computer-Generated Graphic Display of Paraboloidal

Concentrator and Reflected Rays. . . . . . . . . 2-5

3-1. Solar Source Intercept Factors for Paraboloidal

Concentrator with No Surface Error and for a

Perfect Optical System . . . . . . . . . . . . 3-2

3-2. Randomly Perturbed Surface Normal on Concentrator

Surface. . . . . . . . . . . . . . . 3-4

4-1. Computed Model and Experimental Intercept Factors for a

Paraboloidal Concentrator . . . . . . . . . . . 4-2

4-2. Mode1 and Experimental Intercept Factors for a Partially

Complete Concentrator . . . . . . . . . . . . . 4-4 


\section{Figures}

4-3. Projected Solar Source Intecept Factors for a

Paraboloidal Concentrator. . . . . . . . . . . . 4-7

4-4. Solar Source Intercept Factors for Concentrators

with Hypothetical Surface Errors . . . . . . . . . 4-8 


\section{SECTION I}

\section{INTRODUCTION}

This report presents an analysis of solar energy concentrators as optical elements. A substantial amount of experimental and mathematical analyses have already been performed on solar concentrators, but these analyses have generally considered the concentrator as a subsystem component of an overall energy production system. For this reason, characteristics of the concentrator optics have not been systematically analyzed in a manner that is independent of the application.

Any discussion of the operation of a solar concentrator requires a definition of "performance" and a quantitative measure of performance. The basic purpose of a solar concentrator is to image (or focus) solar energy from a large entrance aperture into a small receiver aperture where the radiant energy is converted into thermal energy. The thermal energy is in turn converted to electrical energy by way of a heat engine. The operating efficiency of the thermal-to-electrical energy conversion unit is directly related to the receiver temperature. The temperature of the receiver increases with the mean energy flux density passing into the receiver aperture. The mean energy flux density increases with the image forming quality of the concentrator, i.e., the smaller the image (with respect to the size of the concentrator entrance aperture), the higher the flux density. Herein, the optical performance of a solar concentrator is defined as "the degree to which the concentrator can form a high flux density solar image." Because this report covers only optical imaging, surface reflectance and entrance aperture shading are not included in this definition of performance although these factors do have a significant effect on the overa11 operating performance of a solar concentrator.

The term "concentration ratio," or the ratio of the concentrator aperture area to the receiver aperture area, has often been used to define the optical performance of a solar concentrator. This definition has the disadvantage of being both incomplete and subject to misinterpretation because there is no precise definition of the fraction of the focal plane image that passes through the receiver aperture. For example, a $13 \%$ change in the receiver aperture radius (corresponding to a $30 \%$ change in the concentration ratio) would give a $1 \%$ change in the total input energy.

There are many ways to describe the image forming quality of an optical system. For solar concentrators, a very practical and generally accepted description is the focal plane "intercept factor" curve, which is the same as the standard optical term "encircled energy." These data can be expressed as a table of measured or calculated points that are presented in graphical form. The curve indicates the fract: on of the total image energy passing through an aperture with a radius that is specified by a linear distance from the optical axis in the focal plane or by an angle with the vertex at the center of the concentrator. The latter parameter is dimensionless; as a consequence, the results can be applied to any concentrator regardless of linear dimensions. 
A practical way to measure the image forming characteristics of a reflecting panel or an assembled concentrator is use of a fixed point source of light. A high intensity spotlight at a distance of $1000 \mathrm{ft}$ is ideal in a moderately dark environment, but good measurements can also be made with a high intensity light source at a distance of several focal lengths from the concentrator vertex. The size of the image from an extended source is determined by both the size of the source and the optical imperfections. In this case, it is generally difficult, if not impossible, to make a quantitative determination of the optical imperfections. The final confirmation of concentrator performance must be made with the sun as a source, but for preliminary testing the sun has the disadvantage of both high levels of radiant energy and constant motion.

The intercept factor curve can be directly measured with a photometer and a series of circular apertures. For small panels, a Fresnel lens can be used to image the energy passing through the aperture onto the photometer. For large panels, or an assembled concentrator, an imaging photometer can be used to measure the energy falling outside the aperture of a white annular target. With a series of water-cooled apertures and a cold-water cavity calorimeter, the intercept factor curve can be measured when the concentrator is pointing at the sun. The intercept factor data can also be obtained by the use of a photometer or radiometer that scans the image. Each aperture measurement is divided by the total image measurement to obtain the intercept factor for that aperture. For ray tracing calculations, each point is determined by dividing the number of rays that fall within a specified circle by the total number of rays falling on the concentrator.

An intercept factor curve can describe the image from a point source at a finite or infinite distance, or it can describe the image from the sun. It is also an ideal format for representing both the measured and calculated image data. It can be used to directly determine the collection efficiency of an existing thermal receiver or determine the relationship between receiver temperature and the optimum receiver aperture radius.

Prototype concentrator systems have been built on the basis of overall system analyses and subsequently tested under operating conditions. Difficulties arise when total performance does not meet expectations and there is no direct way to systematically isolate the problems. These problems may result from any or all of the concentrator components: mirror geometry and surface properties, mirror mounting and tracking instabilities, receiver configuration and location, etc.

Because the optical performance of the concentrator is paramount to the success of the energy conversion, a detailed systematic study was made of the optics of a prototype concentrator. 1 This study was based on an interactive

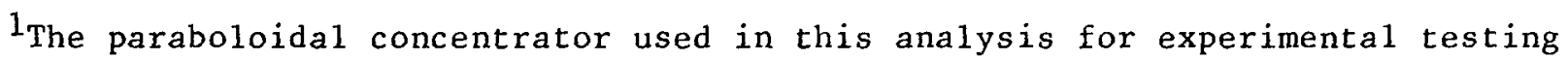
is the 12-m-diameter Parabolic Dish Concentrator No. 1 (PDC-1), which is described in detail in a companion report entitled Development and Testing of Parabolic Dish Concentrator No. 1 by E.W. Dennison and T.O. Thostesen, Jet Propulsion Laboratory, 5105-143, November 1984. 
development of a mathematical model and experimental testing procedures. The experimental procedures used a point source of light at a finite distance and were performed under static mirror conditions. Complete and incomplete mirrors were incorporated into the testing to determine concentrator characteristics early in the manufacturing process and to evaluate quality control procedures.

Previous analyses (Refs. 1 through 4), for example, have incorporated a11 of the system components mentioned into a single comprehensive framework, hence making it difficult to isolate specific effects. Moreover, these analyses are specific to paraboloidal concentrators. The mathematical approach used by these authors emphasizes geometric considerations and utilizes cone optics (an analysis that incorporates the solar disk as an intrinsic part of the computations) as a procedure. This approach is burdened with the problems of transcendental equations that are common to many geometric optical problems. The references cited tend to minimize the requirement for extensive use of computers and their associated expense, and provide procedures for circumventing direct numerical analyses.

The procedures presented here also rely on computed results. With current microcomputing technology, an exact and general formulation can be utilized that is neither disproportionately time-consuming nor expensive and can be made portable enough for use at test site locations. In addition, the procedures are not limited to paraboloidal configurations or single mirror systems. Facetted mirrors, spherical mirrors, and mirrors of special design may be modelled and analyzed with equal facility. 

SECTION II

COMPUTER MODEL

The computer model developed to simulate the concentrator is based on three-dimensional ray tracing. The law of reflection is formulated as a set of vector equations involving the incident rays, reflected rays, and the normal to the concentrator surface at the reflection points. The reflected ray direction is computed for a specified source location, a particular optical surface, and a specified reflection point on the concentrator. Iterating the computation for an array of reflection points and normal vectors suitably describing the surface produces an array of reflected rays.

The points of intersection of these rays with the receiving surface forms a model of the optical image. The coordinates of the intersection points are calculated explicitly, and therefore may be analyzed in the same way that experimental data are analyzed. The number of reflected rays per unit area for prescribed small areas of the image represents the intensity in that area. For intercept factor calculations, the image plane is divided into concentric apertures centered about the optical axis.

In the interest of having a methodical approach to the analysis, the modelling proceeded in a sequence of three distinct steps. The first modelled a perfect geometric concentrator free from surface irregularity and illuminated by a point source. The second introduced variable degrees of surface irregularity into the system, and the third integrated the results of the first two and used as a source an array of points that simulate the finite solar disk.

The vector nature of the three-dimensional reflection problem is illustrated in Figure 2-1 where two reflection points are shown. The rays $\overrightarrow{\mathrm{I}}_{1}$ and $\overrightarrow{\mathrm{I}}_{2}$ are vectors representing incident rays from a single point source. The vectors $\overrightarrow{\mathrm{N}}_{1}$ and $\overrightarrow{\mathrm{N}}_{2}$ are the surface normals particular to the paraboloidal surface with a focal length $f \cdot \vec{R}_{1}$ and $\vec{R}_{2}$ are the resulting reflected rays terminating at the receiving plane.

As indicated in the figure, the law of reflection requires that the angle of incidence and reflection be equal in each case:

$$
\begin{aligned}
& \phi_{1 i}=\phi_{1 r} \\
& \phi_{2 i}=\phi_{2 r}
\end{aligned}
$$

and that the incident, normal, and reflected rays be coplanar. Algorithms suitable for iterative programming can be developed by writing the law of reflection as a combination of vector and scalar products of the vectors involved. 


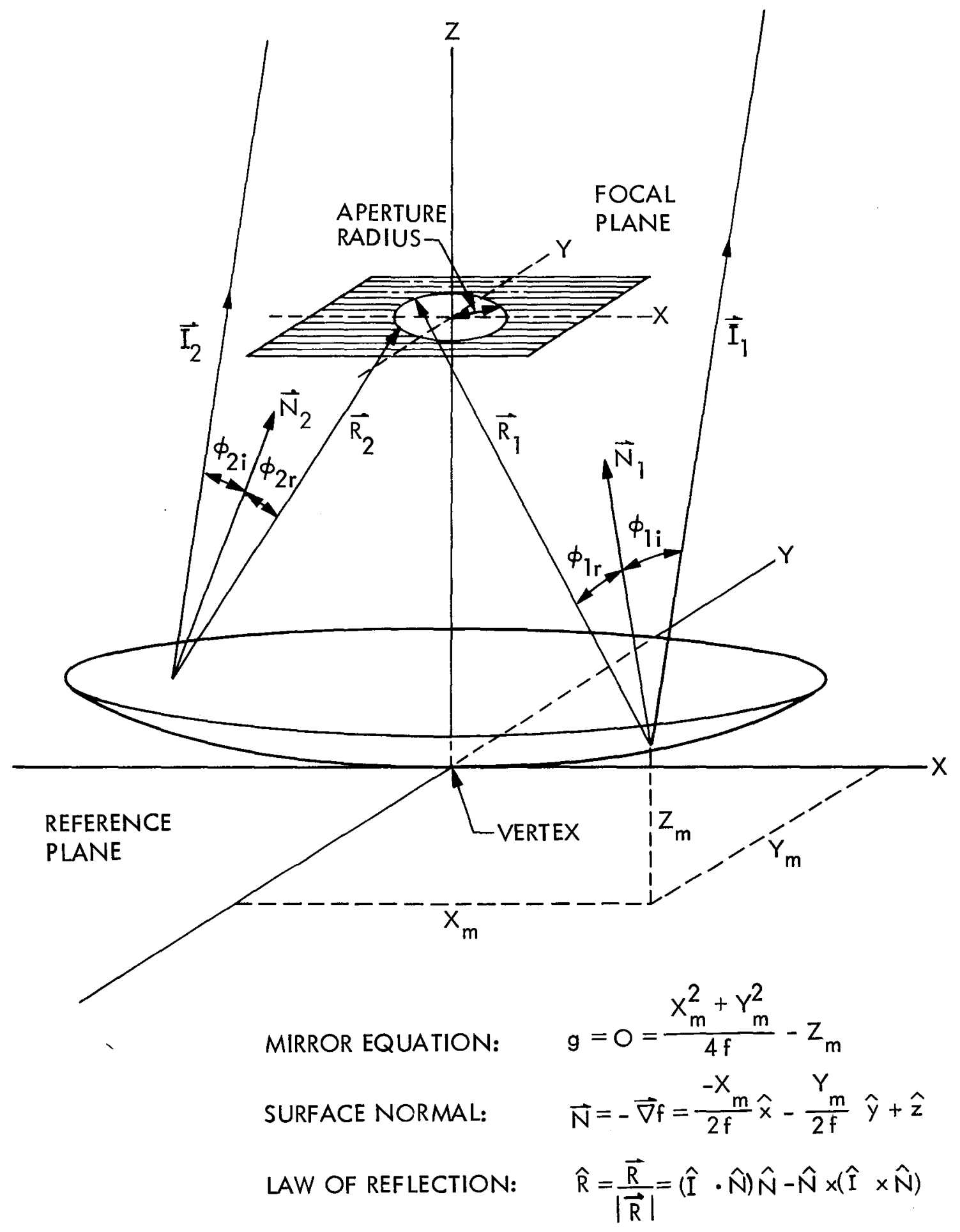

Figure 2-1. Vector Analysis for Three-Dimensional Ray Tracing 
The first step is determining an analytic form for the surface normals. This is accomplished by writing the functional form of the reflecting surface as an equipotential surface and taking the negative gradient of the equation. For the paraboloidal surface with the surface equation usually written as:

$$
\mathrm{Z}_{\mathrm{m}}=\left(\mathrm{X}_{\mathrm{m}}^{2}+\mathrm{Y}_{\mathrm{m}}^{2}\right) / 4 \mathrm{f}
$$

(where the subscript $m$ denotes mirror coordinates relative to the vertex and $f$ is the focal length), the equivalent equipotential is:

$$
\mathrm{g}\left(\mathrm{X}_{\mathrm{m}}, \mathrm{Y}_{\mathrm{m}}, \mathrm{Z}_{\mathrm{m}}\right)=0=\left(\mathrm{X}_{\mathrm{m}}^{2}+\mathrm{Y}_{\mathrm{m}}^{2}\right) / 4 \mathrm{f}-\mathrm{Z}_{\mathrm{m}} \cdot
$$

Application of the negative gradient operator

$$
-\vec{\nabla}=-\left(\partial / \partial X_{m}\right) \hat{x}-\left(\partial / \partial Y_{m}\right) \hat{y}-\left(\partial / \partial Z_{m}\right) \hat{z}
$$

to $g\left(X_{m}, Y_{m}, Z_{m}\right)$ yields the surface normal $\vec{N}$ in terms of the coordinate location $\mathrm{X}_{\mathrm{m}}, \mathrm{Y}_{\mathrm{m}}, \mathrm{Z}_{\mathrm{m}}$ of the reflection point as:

$$
\vec{N}=-\left(\partial g / \partial X_{m}\right) \hat{x}-\left(\partial g / \partial Y_{m}\right) \hat{y}-\left(\partial g / \partial Z_{m}\right) \hat{z}
$$

For a paraboloid this becomes explicitly:

$$
\vec{N}=-\left(X_{m} / 2 f\right) \hat{x}-\left(Y_{m} / 2 f\right) \hat{y}+\hat{z}
$$

The latter equation specifies the components of the surface normal vector for any reflection point.

The incident ray vector from any reflection point to the source becomes

$$
\vec{I}=\left(X_{s}-X_{m}\right) \hat{x}+\left(Y_{s}-Y_{m}\right) \hat{y}+\left(Z_{s}-Z_{m}\right) \hat{z}
$$

where $\mathrm{X}_{S}, \mathrm{Y}_{\mathrm{S}}$, and $\mathrm{Z}_{\mathrm{S}}$ are the coordinates of the point source relative to the vertex. 
With this formulation, both the incident ray and the surface normal are numerically specified. These two vectors are reduced to unit vectors by dividing them by their respective magnitudes:

$$
\begin{aligned}
& \hat{N}=\vec{N} /|\vec{N}| . \\
& \hat{I}=\vec{I} /|\vec{I}| .
\end{aligned}
$$

For a point source at infinity, the incident unit vector can also be described as :

$$
\hat{\mathrm{I}}=\sin \alpha \cos \beta \hat{\mathrm{x}}+\sin \alpha \sin \beta \hat{\mathrm{y}}+\cos \alpha \hat{z},
$$

where $\alpha$ is the radial angle from the optical axis and $\beta$ is the azimuthal angle from the $\mathrm{X}$ axis.

The law of reflection may be written in terms of the scalar (DOT) product $(\hat{I} \cdot \hat{N})$ and the vector (CROSS) product $(\hat{I} \times \hat{N})$ to give the associated reflected ray unit vector as:

$$
\hat{\mathrm{R}}=(\hat{\mathrm{I}} \cdot \hat{\mathrm{N}}) \hat{\mathrm{N}}-\hat{\mathrm{N}} \times(\hat{\mathrm{I}} \times \hat{\mathrm{N}}) \cdot
$$

This unit vector has three components numerically specified in terms of the $\hat{x}$, $\hat{y}$, and $\hat{z}$ directions. These components specify the direction of the reflected ray relative to the coordinate axes.

The vector equation represents three linearly independent component equations that may be written as a matrix multiplication:

$$
\left[\begin{array}{c}
\hat{R}_{x} \\
\hat{R}_{y} \\
\hat{R}_{z}
\end{array}\right]=\left[\begin{array}{ccc}
\left(\hat{N}_{x}^{2}-\hat{N}_{y}^{2}-\hat{N}_{z}^{2}\right) & 2 \hat{N}_{x} \hat{N}_{y} & 2 \hat{N}_{x} \hat{N}_{z} \\
2 \hat{N}_{x} \hat{N}_{y} & \left(\hat{N}_{y}^{2}-\hat{N}_{x}^{2}-\hat{N}_{z}^{2}\right) & 2 \hat{N}_{y} \hat{N}_{z} \\
2 \hat{N}_{x} \hat{N}_{z} & 2 \hat{N}_{y} \hat{N}_{z} & \left(\hat{N}_{z}^{2}-\widehat{N}_{x}^{2}-\widehat{N}_{y}^{2}\right)
\end{array}\right]\left[\begin{array}{c}
\hat{I}_{x} \\
\hat{I}_{y} \\
\hat{I}_{z}
\end{array}\right] .
$$

As a result, the reflection process is viewed mathematically as a transform of the incident ray into the reflected ray with the elements of the transformation depending only on the surface normal components.

The matrix formulation adapts well to iterative computation, and the use of component multiplication eliminates the need for overt use of trigonometric functions. A computer graphic display of the model analogous to Figure 2-1 is shown in Figure 2-2. In the latter figure, the number of zones and the number of reflection points circumscribing each zone are variable. 


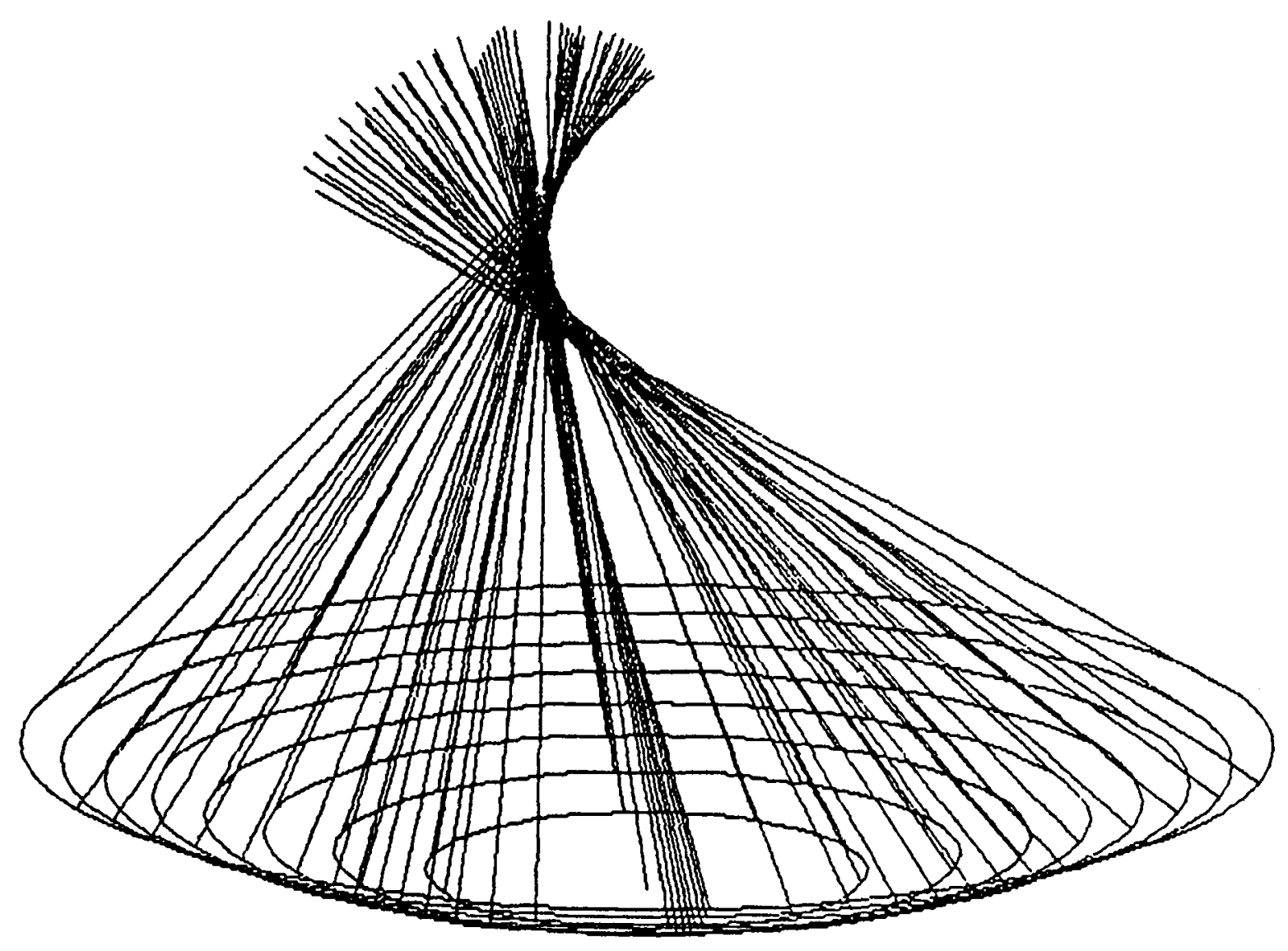

Figure 2-2. Computer-Generated Graphic Display of Paraboloidal Concentrator and Reflected Rays 
The final step in determining the intercept factor is to compute the coordinates of the reflected ray (designated by the subscript p) as it intercepts the target or focal plane. These coordinates can be found from the general equation:

$$
\frac{X_{p}-X_{m}}{\hat{R}_{x}}=\frac{Y_{p}-Y_{m}}{\widehat{R}_{y}}=\frac{Z_{p}-Z_{m}}{\widehat{R}_{z}} .
$$

In practice this becomes two equations:

$$
x_{p}=\left(z_{p}-z_{m}\right) * \frac{\hat{R}_{x}}{\hat{R}_{z}}+x_{m}
$$

and

$$
x_{p}=\left(z_{p}-z_{m}\right) * \frac{\hat{R}_{y}}{\hat{R}_{z}}+Y_{m} .
$$

The coordinates $X_{p}, Y_{p}$, and $Z_{p}$ therefore describe an image point whose distance from the optical axis is:

$$
T=\sqrt{x_{p}^{2}+y_{p}^{2}}
$$

and the ray will be inside the aperture if $T$ is less than the radius of the aperture. The intercept factor is the fraction of the total number of rays striking the concentrator that fall inside the specified focal plane aperture. 


\section{SECTION III}

\section{ABERRATIONAL EFFECTS AND IMPERFECT CONCENTRATORS}

Because the optical source of primary interest for solar concentrators is the sun, a practical optical model must include sources of light that are at least one solar radius from the optical axis. The mean half angle of the solar disk is $4.65 \mathrm{mrad}$, but for convenience a value of $5 \mathrm{mrad}$ has been used for the calculations in this report. For the purpose of the ray tracing model, the points on the sun can be either the angular distance from the optical axis or the $\mathrm{X}, \mathrm{Y}, \mathrm{Z}$ coordinates with respect to the concentrator vertex. (The $\mathrm{Z}$ distance of the sun is approximately $1.5 \times 10^{11} \mathrm{~m}$. )

For a perfect paraboloid, off-axis source points are not formed into point images. These aberrations increase with the angular distance of the point source from the optical axis, and the composite effect of these aberrations is significant. To demonstrate this, an intercept factor curve was calculated for a paraboloidal concentrator with a focal length/diameter $(f / D)$ of 0.5 and a circular source of 5 mrad radius. The results are shown in Figure $3-1$, and the data are given in Table $3-1.2$ For comparison, the intercept factor curve for a perfect optical system (no aberrations) with the same source is also included. The intercept factor is shown as a function of the aperture radius divided by the focal length. This dimensionless parameter is the tangent of the aperture radius angle as viewed from the vertex. Because the angles are small and to clarify the interpretation of the curves, the angle is given in milliradians (mrad).

The intercept factor curve for a perfect paraboloid is the upper 1imit of optical performance. Physical imperfections that extend the intercept factor must be accounted for in any acceptable model. The model described here assumes the uncertainties to be of two categories: systematic and random.

The systematic uncertainties include errors in the geometric form, focal length, and tracking. These errors can be modelled by specifying a different geometry for the mirror and by offsetting the solar source from the optical axis. However, for the PDC-1 concentrator, these errors were detected and corrected by other direct methods and were eliminated as a major concern in the final calculations.

Of more relevance to the model is a technique for simulating the random surface irregularities that are an inherent part of any concentrator. Because the sources of these irregularities are diverse and usually cannot be specifically isolated, a computational scheme based on random surface errors was developed and incorporated into the model. The test of such a modelling technique is comparison with experimental data.

The model is based on the concept that the surface normals are randomly perturbed, and these perturbations cause errors in the concentrator image.

2Data tables are given in the Appendix. 


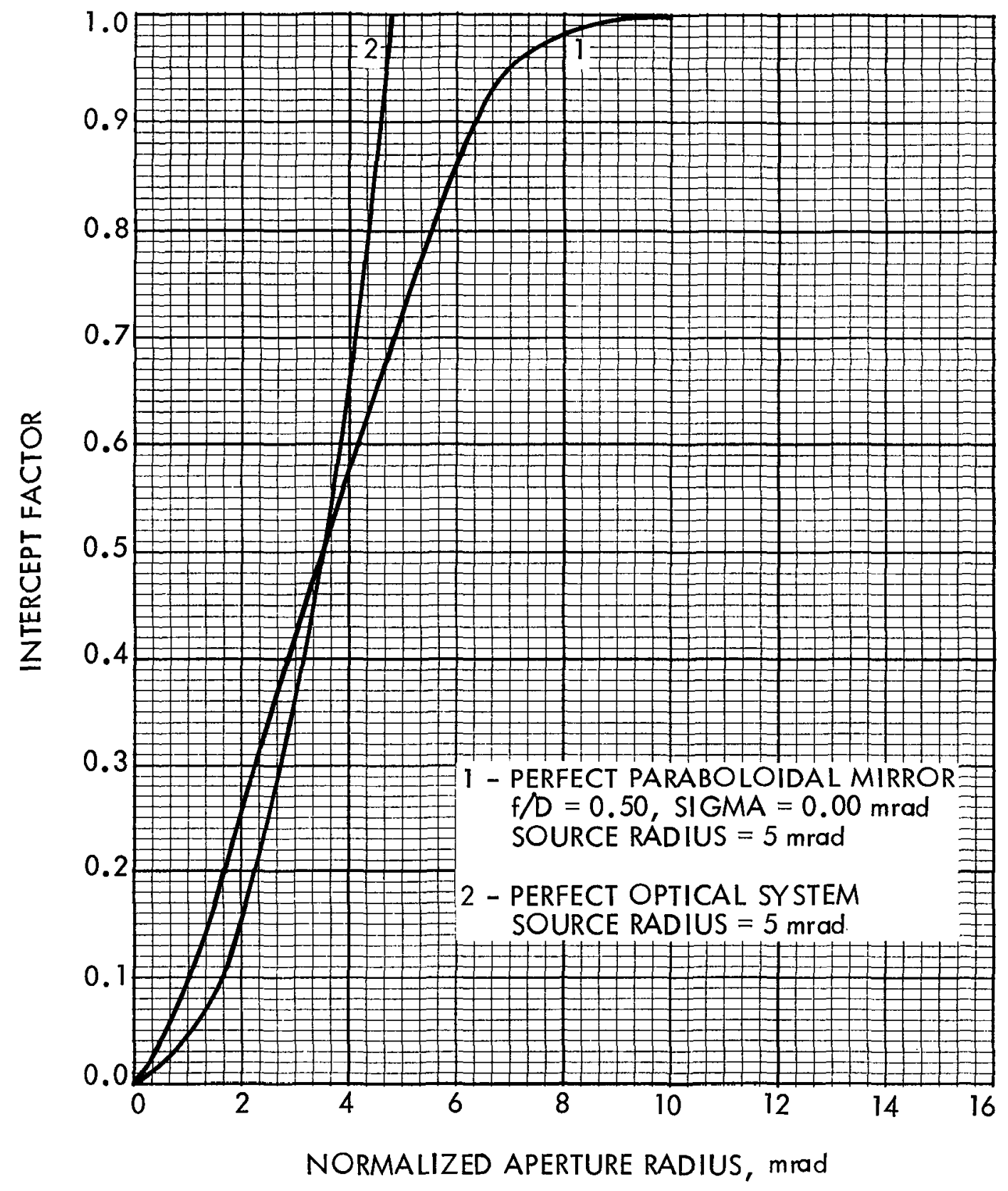

Figure 3-1. Solar Source Intercept Factors for Paraboloidal Concentrator with No Surface Error and for a Perfect Optical System 
Because the concentrator has a large number of small independent surface irregularities, the model must be calculated for a large number of reflection points. The perturbations introduced into the model surface normals were randomly selected but modulated by a Gaussian distribution.

The process is illustrated in Figure $3-2$. In the figure, $\vec{N}$ is the surface normal prescribed by the geometry of the concentrator, and $\vec{N}^{\prime}$ is the perturbed normal. $\vec{N}^{\prime}$ is inclined relative to $\vec{N}$ by an angle $\phi$ and then allowed to precess about $\vec{N}$ with a precession angle $\delta$. The inclination angle $\phi$ is chosen at random from a Gaussian distribution according to the equation:

$$
\operatorname{RDM} 1=[1 /(\sigma \sqrt{2 \pi})] \exp \left(-\phi^{2} / 2 \sigma^{2}\right)
$$

RDM1 is a random number in the range $0<\mathrm{RDM} 1 \leqq 1$. The standard deviation, sigma, is determined empirically by comparison with experimental data and becomes the surface quality factor of the concentrator.

For computational purposes, the equation is inverted to:

$$
\phi=\sigma \sqrt{2}[\ln (1 / \sigma \sqrt{2 \pi})-\ln (\mathrm{RDM} 1)]^{1 / 2} .
$$

The precession angle is selected from a uniform distribution with $\delta=$ $(\operatorname{RDM} 2) \cdot(2 \pi)$ and $0<(R D M 2) \leqq 1$.

The random numbers RDM1 and RDM2 are obtained by separate selections from a random number generator to avoid any coupling of the calculations. Establishing the angles $\phi$ and $\delta$ permits the components of the perturbed normal to be written as those of a vector $\overrightarrow{\mathrm{N}}^{\prime}$ in a coordinate system that has been rotated relative to the vertex axes. A rotation transformation is required to establish these components in the $\hat{x}, \hat{y}, \hat{z}$ coordinate system. 


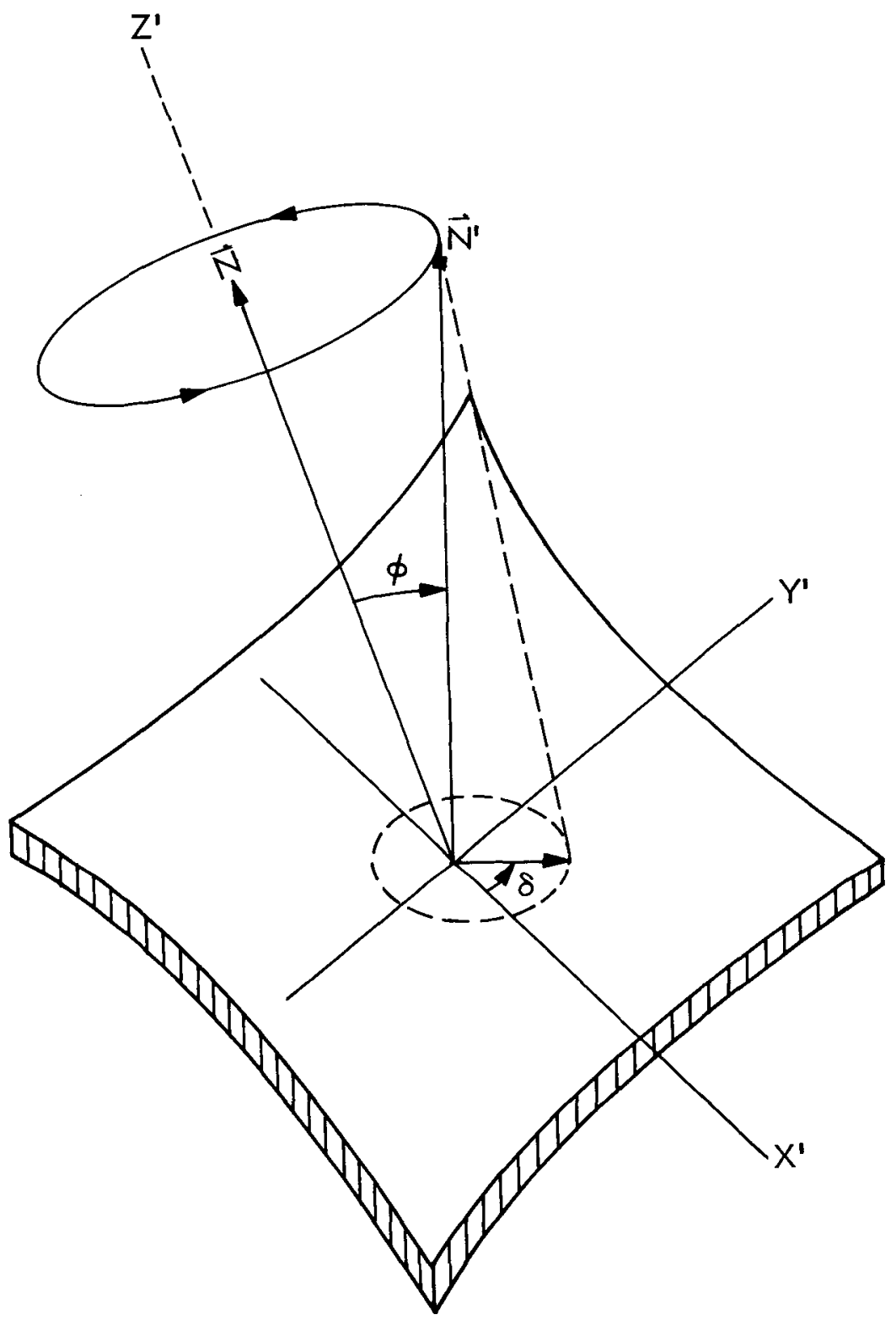

Figure 3-2. Randomly Perturbed Surface Normal on Concentrator Surface 
SECTION IV

RESULTS AND DISCUSSION

As a test of the computer model and of the general procedure, comparisons were made between the model predictions and experimental. measurements for a paraboloidal concentrator. This comparison was designed for three specific objectives: (1) to determine if the modelling procedure had the credibility and sensitivity required for a practical analytic device, (2) to determine a surface quality factor representative of a specific concentrator, and (3) to present an accurate extrapolation from the point source test data to the solar performance.

The concentrator under study had nominal values of $6.00 \mathrm{~m}$ for the focal length and 0.50 for the focal length/diameter ratio ( $f / D)$, and for testing purposes was illuminated with a point source. Experimentally measured intercept factors were compared with those predicted by the computer model.

The experimental procedures developed for using point sources at finite distances permitted direct methodical comparison of intercept factors without the necessity of relying on solar models in the analysis. The sun, as a finite source, enlarges the image and obscures the image defects resulting from concentrator imperfections. The use of point sources permits an accurate analysis of the concentrator and allows the experiment to be well defined. The effect of the finite solar source can be calculated after the optical characteristics of the concentrator have been determined.

\section{A. FULL MIRROR COMPARISONS}

Experimental data for the complete concentrator were gathered with the point source (a high quality spotlight) on the optical axis $900 \mathrm{~m}$ (nominally 150 focal lengths) from the mirror vertex. The image plane was established as the location of minimum image size and was located $6.03 \mathrm{~m}$ from the vertex, very near the nominal focal plane. The intercept factors were determined by measuring the amount of light falling on a series of white apertures. The measurements were made with an imaging photometer mounted at the concentrator vertex.

The subsequent analysis permitted relatively direct comparison with the model results. In the ray trace modelling procedures, the intensity is interpreted as the percentage of rays falling within a circular aperture in the image plane.

The computer model was used in an interactive mode to yield the intercept factor curve shown in Figure 4-1 (data shown in Table 4-1), and established a surface error sigma value of $16.25 \mathrm{mrad}$. Simultaneously, the real focal length was confirmed to be $6.00 \mathrm{~m}$. The RMS (root mean square) deviation of the curve from the data is approximately $1 \%$. It should be noted, however, that the model curve is not an attempt to fit the data parametrically, 


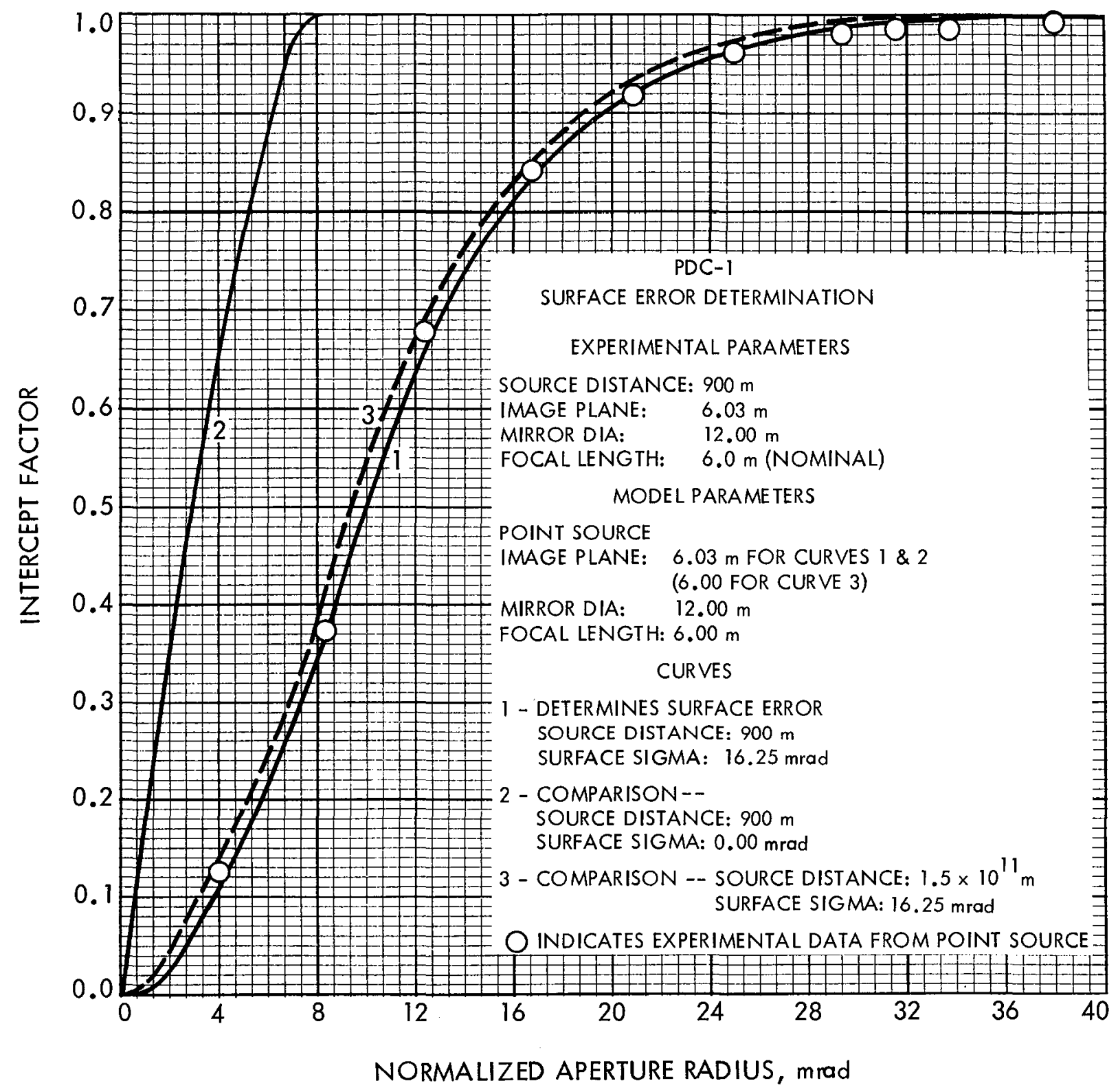

Figure 4-1. Computed Model and Experimental Intercept Factors for a Paraboloidal Concentrator. (Image derived from a point source $900 \mathrm{~m}$ from the vertex.) 
but to establish a surface error number that represents the experimental intercept factors. The criteria for the best representation was based on subjective judgment rather than a numeric RMS deviation.

At the beginning of the testing program, there was some concern about the propriety of using a point source at a finite distance because a paraboloidal concentrator gives a well defined image only for an infinitely distant point source. In practice, this did not present a problem because an accurate model could simulate any test configuration.

The comparisons between numerous model intercept factors and those for the experiment did demonstrate that the surface error was the only unknown parameter. For that reason, the nominal values for the focal length, mirror diameter, image plane distance, and source distance could be used directly in the final model.

The development of the various computer models necessary to establish the comparative intercept factors provided valuable information concerning the sensitivity of the model to the physical parameters. In particular, the intercept factor is relatively insensitive to the $\mathrm{f} / \mathrm{D}$ ratio.

Precise measurement of the experimental image plane position and source distance are crucial to the comparisons, as is a reasonably accurate nominal value for the focal length. The most sensitive factor in the modelling is the relative focal point/image plane distance because of the low $\mathrm{f} / \mathrm{D}$ ratio of the concentrator. The size of this image changes rapidly with small displacements of the intercept plane away from the focal point.

Variation of other parameters in addition to the surface error to obtain reasonable mode1-experiment agreement can be done, but involves looking for subtle changes in the shape of the intercept factor curve as well as in its overall magnitude. For this reason, such comparison depends heavily on the precision and quantity of experimental data. The excellent agreement of the computed data with the experimental data indicated that changes to the nominal parameters were unnecessary.

\section{B. POINT SOURCE TESTING OF INCOMPLETE MIRRORS}

The feasibility of providing mirror quality information during the manufacturing of the concentrator panels was investigated by performing point source testing with only four of the twelve panels. The testing procedures were similar to those used for the complete concentrator, i.e., a point source at $900 \mathrm{~m}$ was used to illuminate the mirror, and the image was photometrically measured to determine the intercept factor.

The experimental and computer model data are shown in Figure 4-2 and Table 4-2. The best comparison for the four-panel system data yielded a surface quality sigma value of $14.0 \mathrm{mrad}$ as the aggregate for the four panels. The computer model treated the data as though it were for a complete concentrator (permissible because of the rotational symmetry) in order to determine which of the physical parameters required adjustment. Combinations 


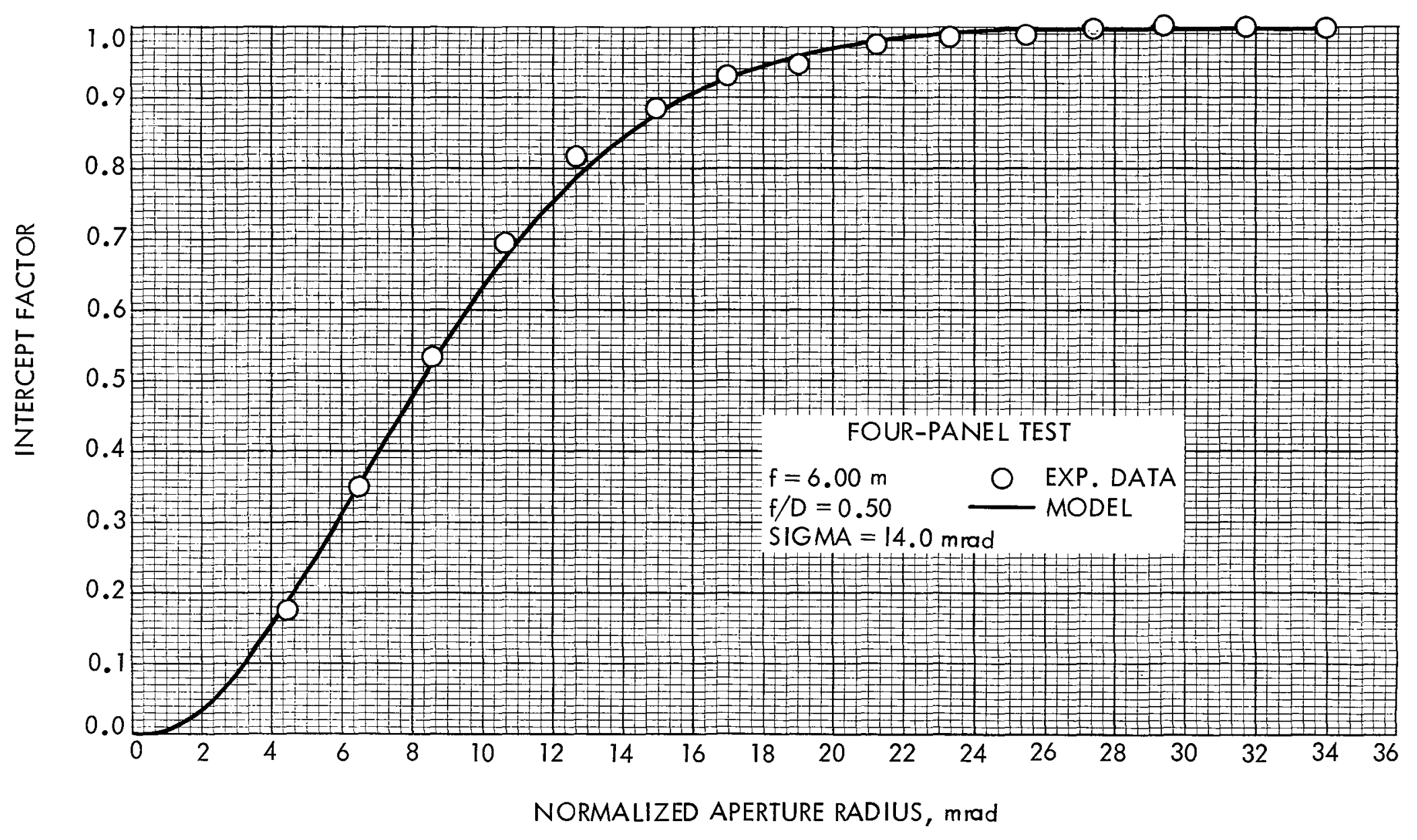

Figure 4-2. Model and Experimental Intercept Factors for a Partially Complete Concentrator. (Image derived from a point source $900 \mathrm{~m}$ from mirror vertex.) 
of focal length, image plane, diameter, and surface error were modelled. The study provided insight into the sensitivity of the modelling procedures to parametric changes, as well as quantitative data concerning the concentrator.

Two different approaches to the parametric search yielded independent, reasonable representations of the experimental intercept factors. The first made the assumption that the effective focal length was $6.0 \mathrm{~m}$, the focal length/diameter ratio was 0.50 , and the point source at $900 \mathrm{~m}$ was close enough to infinity to assume imaging at $6.0 \mathrm{~m}$. No acceptable intercept factor was found for these parameters. An acceptable intercept factor agreement was obtained by changing the $f / D$ ratio to 0.54 and by using a surface error sigma of $12.7 \mathrm{mrad}$. However, this would imply a mirror diameter of $11.1 \mathrm{~m}$ instead of the actual $12 \mathrm{~m}$. Moreover, modelling a point source at infinity with these parameters did not give an intercept factor similar to that of the 900-m data.

The second model was constructed to conform as closely as possible to the actual experimental measurements. The focal length and $f / D$ were reestablished at $6.00 \mathrm{~m}$ and 0.50 , respectively, but the image plane was set at $6.03 \mathrm{~m}$ as was actually measured instead of at the previously assumed $6.0 \mathrm{~m}$. These parameters yielded the best comparison and the sigma of $14.0 \mathrm{mrad}$. These parameters, when modelled for a point source at infinity, give an intercept factor showing the same relationship to the $900-\mathrm{m}$ data as the one shown in Figure 4-1 for the complete concentrator. This is the relationship expected of correct modelling.

These studies of the four-panel assembly reinforce the importance of accurately determining the image plane/focal plane distance for point source testing and of accurately measuring the test configuration. This work also provides the basis for further investigation of techniques for analyzing concentrator optical elements early in their construction.

The difference between the surface quality of the four-panel system and that of the full mirror is not large, and such an increase in surface irregularity is not unexpected as additional panels are installed. Because the computer model can be easily tailored to specific parameters and the experimental measurements can be made within reasonable physical constraints, this modelling method is a promising mode of quality control. Further study of this application is clearly merited.

\section{INTERCEPT FACTOR COMPARISONS WITH ONE PARAMETER}

The ability to obtain good model-experiment intercept factor comparisons using only one parameter, sigma, as the adjustable parameter should not be surprising because the computation of the surface error actually incorporates two variables: (1) the tilt angle of the perturbed normal relative to the ideal value and (2) the precession angle of the tilted normal. Both parameters are called independently from a random number generator. The first assumes a Gaussian modulation of the random numbers and has a standard deviation available as an adjustable parameter. The second assumes a uniform distribution of the precession angles. As a result, the second parameter has 
no standard deviation and does not appear explicitly. The inclusion of the two independent parameters does introduce, however, both azimuthal and radial effects of surface irregularities and, thus, gives a reasonable representation of the actual optical surface.

\section{SOLAR EXTRAPOLATION}

The model can project the concentrator solar performance as a direct extrapolation of the test results. Assuming constant geometric factors for the mirror and the surface quality factor to be sigma $=16.25 \mathrm{mrad}$, intercept factors for a solar source were modelled. For this specific extrapolation, the simplest solar model, i.e., that of a uniformly illuminated disk, was used.

The solar model was given rotational symmetry about the optic axis, an angular radius of $5.0 \mathrm{mrad}$, and a source distance of $1.5 \times 10^{11} \mathrm{~m}$. This was accomplished by assuming that the source rays are on a 1 ine extending from the optical axis outward to $5.0 \mathrm{mrad}$ in $1.0 \mathrm{mrad}$ increments. Each point was the source of 3565 rays distributed uniformly over the surface of the concentrator for a total of 21,390 rays. The rays are intercepted at the focal plane $(6.00 \mathrm{~m})$. The resulting solar source intercept factor is shown in Figure 4-3. The corresponding data are given in Table 4-3.

Because the surface error $(16.25 \mathrm{mrad})$ is significantly greater than the solar radius of $5 \mathrm{mrad}$ and therefore dominates the image degradation, the intercept factor of the sun is not greatly different from that of a single point source on axis.

It should be noted with caution that if the solar radius is attributed a sigma value of $\sigma_{s}=5 \mathrm{mrad}$ and combined with the surface error $\sigma_{\mathrm{m}}=16.25 \mathrm{mrad}$ to give a total of $\sigma_{\mathrm{T}}=\left(\sigma_{\mathrm{m}}^{2}+\sigma_{\mathrm{s}}^{2}\right) 1 / 2=17.0 \mathrm{mrad}$, a point source illuminating a mirror with a surface error of $\sigma_{\mathrm{m}}=17.0 \mathrm{mrad}$ gives an intercept factor essentially identical to that of the solar source with $\sigma_{m}=16.25 \mathrm{mrad}$. Although this is not unexpected when $\sigma_{m} \gg \sigma_{s}$, caution must be used in its general application. The same procedure using $\sigma_{\mathrm{m}}=14.7 \mathrm{mrad}$ and $\sigma_{\mathrm{s}}=5 \mathrm{mrad}$ does not produce acceptable results even though the intercept factors are similar. Conversely, when $\sigma_{m} \ll \sigma_{s}$, the intercept factor is primarily determined by the $f / D$ ratio of the concentrator and the angular size of the sun and is not dependent on the surface errors.

For comparison, Figure 4-4 shows the intercept factors for a 5-mrad, uniformly radiating solar disk and hypothetical mirror surfaces with sigma values of $11,13,15$, and $17 \mathrm{mrad}$.

In the intercept region of 85 to $95 \%$, the curves indicate that an uncertainty of $2 \mathrm{mrad}$ in the initial determination of the surface error could lead to errors as large as $10 \%$ in the projected solar performance. However, the quality of the experimental data available indicates that the surface error can be ascertained to within $+0.25 \mathrm{mrad}$. The intercept factor curves show that the latter value would translate to a projected intercept factor uncertainty of $2 \%$ or less. Improved data acquisition methods would probably reduce this uncertainty. 


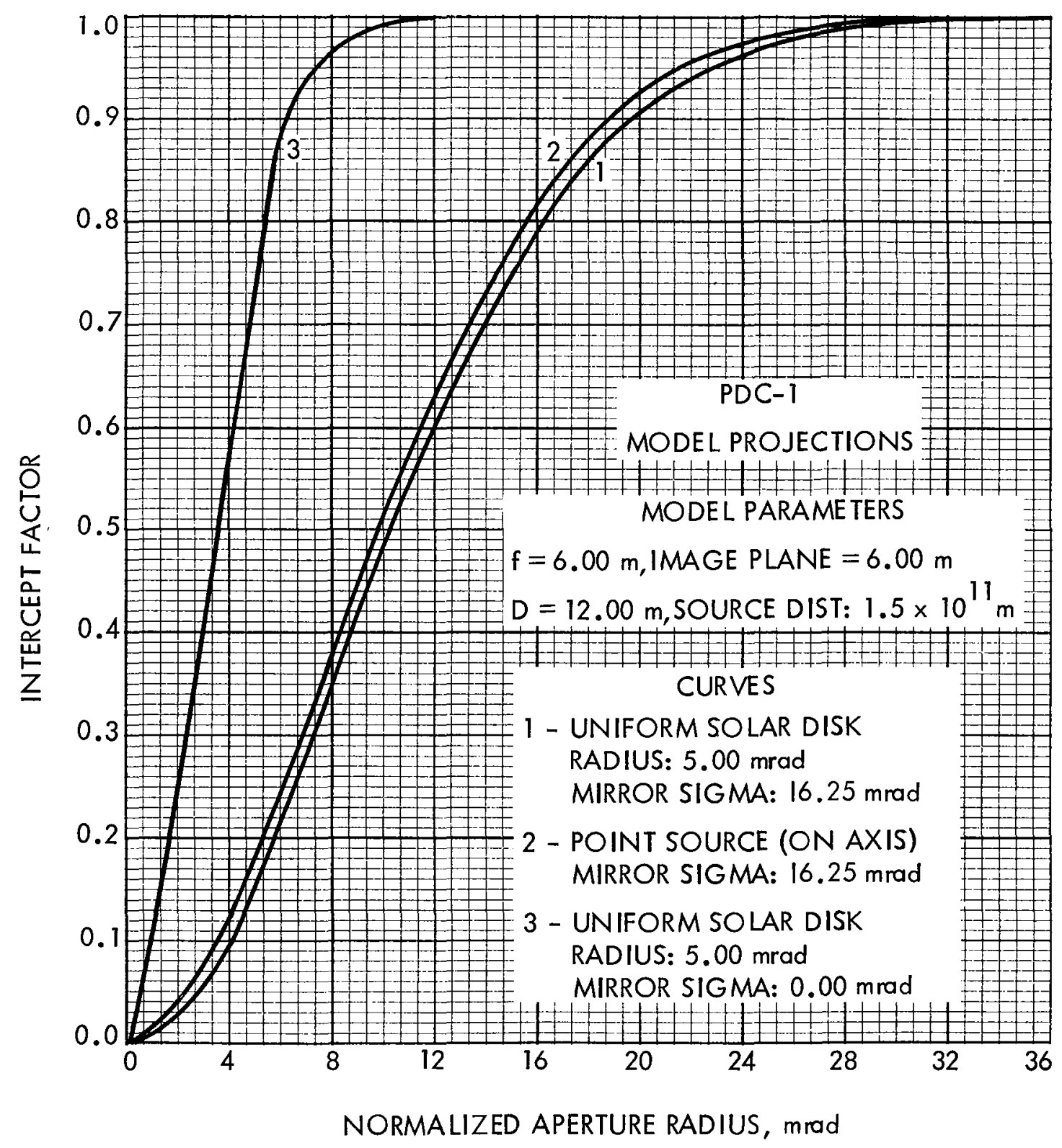

Figure 4-3. Projected Solar Source Intercept Factors for a Paraboloidal Concentrator 


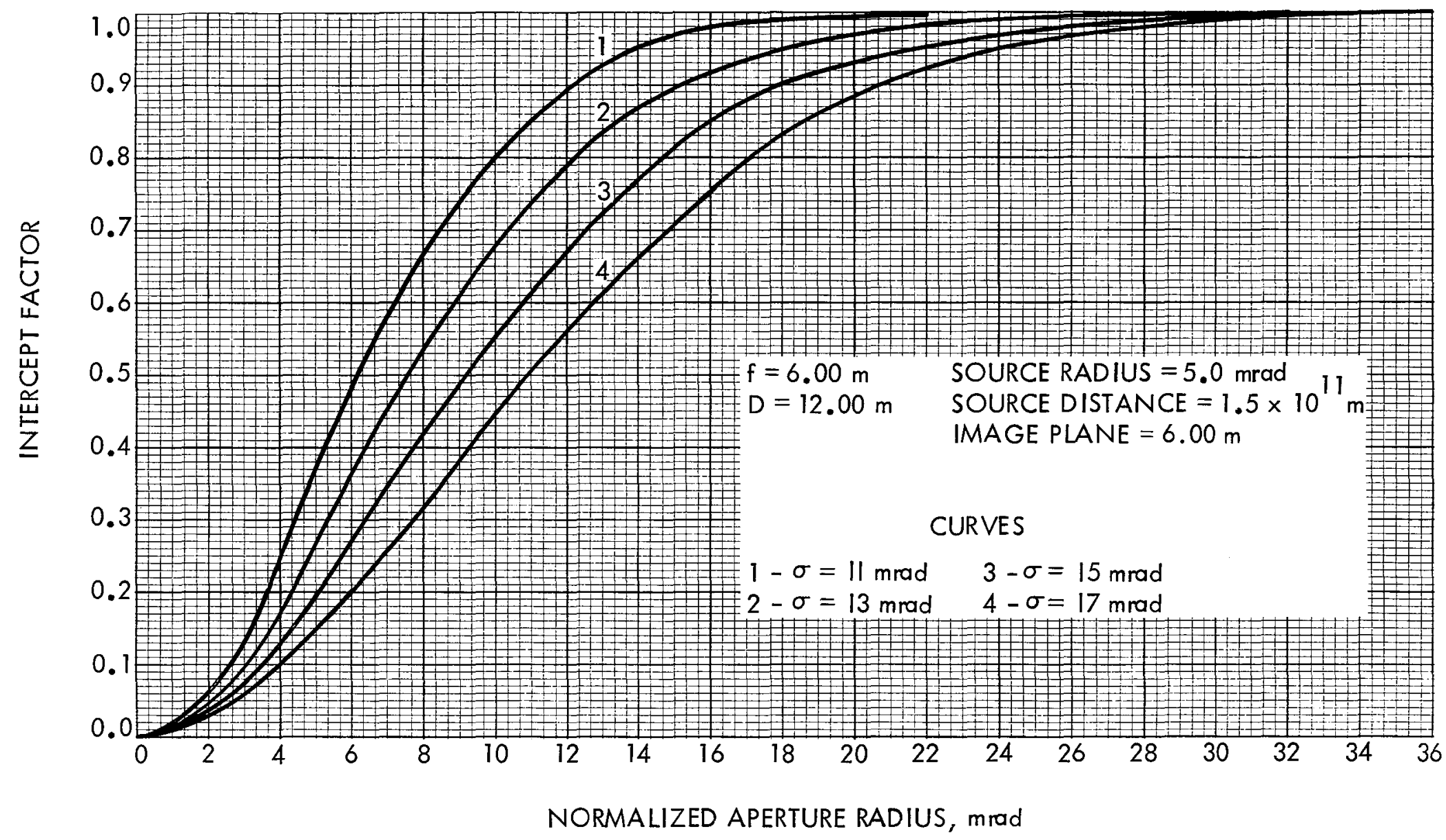

Figure 4-4. Solar Source Intercept Factors for Concentrators with Hypothetical Surface Errors 
It should be noted that the measurements were made under static mirror conditions. During actual operation with the concentrator tracking the sun, uncertainties in tracking position and the changing gravity load could affect the total performance. Measurements of this type can be made with cold-water calorimeters, but are generally time-consuming and limited to a few aperture values. 


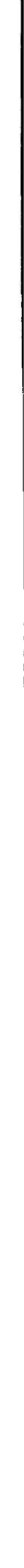




\section{CONCLUSIONS}

The use of basic paraboloidal geometry with the $f / D$ ratio and a single surface or slope error as the fundamental parameters to describe a solar concentrator is common to both this work and that of previous authors. It is reassuring to note that these different approaches give similar results.

The problem with previous solar concentrator models is that they cannot be used for simple testing of single optical panels or non-solar tests of assembled concentrators. This problem has been resolved by the use of the intercept factor curve with a point source of light at any distance from the concentrator or with the sun as a source. The intercept factor curve can be used to describe the results of a mathematical model of a concentrator or to represent reduced data from experimental image measurements. Measurements can be made by scanning a photodetector or flux mapper over the image or by the use of an integrating photometer or calorimeter to measure the relative intensity of the image falling inside a series of circular apertures. With the sun as a source, these intercept factor curves can be used to evaluate the performance of power conversion thermal receivers.

The use of ray tracing computer programs as described herein is both powerful and practical. These programs can include any type of optical configuration from simple paraboloids to arrays of spherical facets. These programs can be adapted to microcomputers at an acceptable cost in operating time. The sophisticated graphics displays now available on many microcomputing systems can be used for real-time interactive comparison of calculated and measured data.

When the optical testing of the JPL PDC-1 solar concentrator began, there was a clear need for a comprehensive method to handle both the theoretical and experimental aspects of imaging characteristics of solar concentrators. While the work described in this report is not definitive, it does demonstrate that the use of ray tracing programs and intercept factor curves can provide a practical way to fulfill this need. 


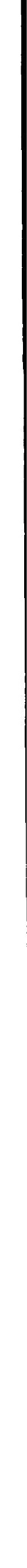


1. Wen, L., Huang, L., Poon, P., and Carley, W., "Comparative Study of Solar Optics of Paraboloidal Concentrators," ASME, 79-WA/Sol-8, 1979.

2. Wen, L., "Effects of Optical Surface Properties on High Temperature Solar Thermal Energy Conversion," Journal of Energy, Vol. 3, No. 2, 1979.

3. O'Neill, M.J., and Hudson, S.L., "Optical Analysis of Paraboloidal Solar Concentrators," Proceedings of the 1978 SPIE Annual Meeting, Vol. 2, 1978, pp. 855-862.

4. Dendt, P., and Rabl, A., "Optical Analysis of Point Focus Paraboloidal Radiation Concentrators," App1ied Optics, Vo1. 20, 1981, pp. 674-683. 


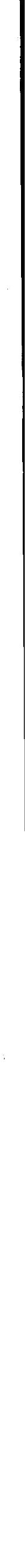


APPENDIX

DATA TABLES FOR FIGURES IN TEXT

Table 3-1. Model Computed Intercept Factor Data for Figure 3-1. . . . A-3

Table 4-1. Model Computed and Experimental Data for Figure 4-1. . . . A-4

Table 4-2. Mode1 Computed and Experimental Data for Figure 4-2. . . A-8

Table 4-3. Mode1 Computed Solar Source Projections for Figure 4-3 . . . A-10 

Table 3-1. Model Computed Intercept Factor Data for Figure 3-1.

(Solar Source Intercept Factor; Perfect Paraboloidal Mirror; $f / D=0.50$; Mode1 Sigma $=0.00 ;$ Source Distance $=1.5 \times 10^{11}$;

Source Radius $=5.0 \mathrm{mrad}$ )

\begin{tabular}{|c|c|c|}
\hline \multirow[b]{2}{*}{$\begin{array}{c}\text { Normalized } \\
\text { Aperture Radius, } \\
\text { mrad }\end{array}$} & \multicolumn{2}{|c|}{ Intercept Factor } \\
\hline & $\begin{array}{l}\text { Perfect } \\
\text { Paraboloidal } \\
\text { Mirror }\end{array}$ & $\begin{array}{l}\text { Perfect } \\
\text { Optica1 } \\
\text { System }\end{array}$ \\
\hline 0.6 & 0.042 & 0.014 \\
\hline 0.8 & 0.085 & 0.026 \\
\hline 1.0 & 0.099 & 0.040 \\
\hline 1.2 & 0.142 & 0.058 \\
\hline 1.4 & 0.170 & 0.078 \\
\hline 1.6 & 0.199 & 0.102 \\
\hline 1.8 & 0.236 & 0.130 \\
\hline 2.0 & 0.262 & 0.160 \\
\hline 2.2 & 0.297 & 0.194 \\
\hline 2.4 & 0.327 & 0.230 \\
\hline 2.6 & 0.359 & 0.270 \\
\hline 2.8 & 0.392 & 0.314 \\
\hline 3.0 & 0.419 & 0.360 \\
\hline 3.5 & 0.498 & 0.490 \\
\hline 4.0 & 0.578 & 0.640 \\
\hline 4.5 & 0.656 & 0.810 \\
\hline 5.0 & 0.711 & 1.000 \\
\hline 5.5 & 0.814 & \\
\hline 6.0 & 0.873 & \\
\hline 6.5 & 0.915 & \\
\hline 7.0 & 0.943 & \\
\hline 7.5 & 0.963 & \\
\hline 8.0 & 0.977 & \\
\hline 8.5 & 0.986 & \\
\hline 9.0 & 0.992 & \\
\hline 9.5 & 0.996 & \\
\hline 10.0 & 0.999 & \\
\hline
\end{tabular}


Table 4-la. Model Computed Data for Figure 4-1

(Date: 11/11/83; Geometry: Modified Parabola; Foca1 Length $=6.00$; $\mathrm{f} / \mathrm{D}$ Ratio $=0.500$; 非 Rays $=3565$; 非 Circles $=100$; 非 Points $/$ Circle $=36$;

Plane Cut Z; Value: 6.030; Sigma $=16.25$;

Source Points: $X=0, Y=0, Z=900$ )

\begin{tabular}{|c|c|c|}
\hline $\begin{array}{l}\text { Normalized Aperture } \\
\text { Radius, mrad }\end{array}$ & $\begin{array}{l}\text { Intercept } \\
\text { Factor }\end{array}$ & $\begin{array}{l}\text { Points } \\
\text { Out }\end{array}$ \\
\hline 2.00 & 0.022 & 3485 \\
\hline 4.00 & 0.105 & 3190 \\
\hline 4.21 & 0.119 & 3142 \\
\hline 6.00 & 0.225 & 2762 \\
\hline 8.00 & 0.356 & 2297 \\
\hline 8.42 & 0.394 & 2159 \\
\hline 10.00 & 0.492 & 1812 \\
\hline 12.00 & 0.624 & 1339 \\
\hline 12.64 & 0.660 & 1212 \\
\hline 14.00 & 0.721 & 995 \\
\hline 16.00 & 0.810 & 679 \\
\hline 16.85 & 0.831 & 601 \\
\hline 18.00 & 0.863 & 488 \\
\hline 20.00 & 0.912 & 314 \\
\hline 21.06 & 0.932 & 244 \\
\hline 22.00 & 0.948 & 186 \\
\hline 24.00 & 0.964 & 127 \\
\hline 25.27 & 0.972 & 100 \\
\hline 26.00 & 0.976 & 86 \\
\hline 28.00 & 0.985 & 53 \\
\hline 29.49 & 0.990 & 35 \\
\hline 30.00 & 0.992 & 29 \\
\hline 31.59 & 0.994 & 20 \\
\hline 32.00 & 0.996 & 16 \\
\hline 33.70 & 0.998 & 6 \\
\hline 34.00 & 0.998 & 6 \\
\hline 36.00 & 0.999 & 4 \\
\hline 37.91 & 0.999 & 4 \\
\hline 38.00 & 0.999 & 3 \\
\hline 40.00 & 0.999 & 3 \\
\hline 42.00 & 0.999 & 2 \\
\hline 42.12 & 0.999 & 2 \\
\hline 44.00 & 1.000 & 0 \\
\hline
\end{tabular}


Table 4-1b. Mode1 Computed Data for Figure 4-1

(Date: 11/11/83; Geometry: Modified Parabola; Focal Length $=6.00$; $\mathrm{f} / \mathrm{D}$ Ratio $=0.500 ;$ 非 Rays $=3565$; 非 Circles $=100$; 非 Points/Circle = 36;

Plane Cut $\mathrm{Z}$; Value: 6.000; Sigma $=16.25$;

Source Points: $X=0, Y=0, Z=1.5 \times 10^{11}$ )

Normalized Aperture Radius, mrad

\section{Intercept}

Factor
Points

Out

3437

3122

2683

2199

1729

1289

947

651

451

305

182

110

0.969

0.983

0.990

0.996

0.998

0.999

0.999

0.999

1.000
35

13

32.0

34.0

36.0

38.0

40.0 
Table 4-1c. Mode1 Computed Data for Figure 4-1

(Date: 11/10/83; Geometry: Modified Parabola; Focal Length $=6.00$; $\mathrm{f} / \mathrm{D}$ Ratio $=0.500$; 非 Rays $=3565$; 非 Circles $=100 ;$ 非 Points/Circle $=36$; Plane Cut Z; Value: 6.030; Sigma $=0.0000$;

Source Points: $X=0, Y=0, Z=900$ )

\begin{tabular}{ccc}
$\begin{array}{c}\text { Normalized Aperture } \\
\text { Radius, mrad }\end{array}$ & $\begin{array}{c}\text { Intercept } \\
\text { Factor }\end{array}$ & $\begin{array}{c}\text { Points } \\
\text { Out }\end{array}$ \\
\hline 0.50 & 0.061 & 3448 \\
1.00 & 0.172 & 2952 \\
1.50 & 0.273 & 2592 \\
2.00 & 0.364 & 2268 \\
2.50 & 0.444 & 1980 \\
3.00 & 0.525 & 1692 \\
3.50 & 0.596 & 1440 \\
4.00 & 0.657 & 1224 \\
4.50 & 0.717 & 1008 \\
5.00 & 0.778 & 792 \\
5.50 & 0.828 & 612 \\
6.00 & 0.879 & 432 \\
6.50 & 0.929 & 252 \\
7.00 & 0.980 & 72 \\
7.50 & 1.000 & 0 \\
\hline
\end{tabular}


Table 4-1d. Experimental Data for Figure 4-1

(Fu11 Mirror Experimenta1 Data Source: Point, Visible Source Distance: $900 \mathrm{~m}$; Image Plane: $6.03 \mathrm{~m}$ )

\begin{tabular}{|c|c|c|c|}
\hline $\begin{array}{l}\text { Aperture } \\
\text { in. }\end{array}$ & $\begin{array}{r}\text { Radius } \\
\mathrm{mm}\end{array}$ & $\begin{array}{l}\text { Normalized Aperture } \\
\text { Radius, mrad }\end{array}$ & $\begin{array}{c}\text { Intercept Factor } \\
\text { Measured }\end{array}$ \\
\hline 1.000 & 25.4 & 4.21 & 0.131 \\
\hline 2.000 & 50.8 & 8.42 & 0.377 \\
\hline 3.000 & 76.2 & 12.64 & 0.670 \\
\hline 4.000 & 101.6 & 16.85 & 0.839 \\
\hline 5.000 & 127.0 & 21.06 & 0.927 \\
\hline 6.000 & 152.4 & 25.27 & 0.965 \\
\hline 7.000 & 177.8 & 29.49 & 0.981 \\
\hline 7.500 & 190.5 & 31.59 & 0.985 \\
\hline 8.000 & 203.2 & 33.70 & 0.988 \\
\hline 9.000 & 228.6 & 37.91 & 0.991 \\
\hline 10.000 & 254.0 & 42.12 & 0.994 \\
\hline 11.000 & 279.4 & 46.33 & 0.997 \\
\hline 12.000 & 304.8 & 50.55 & 0.998 \\
\hline
\end{tabular}


Table 4-2a. Mode1 Computed Data for Figure 4-2

(Date: 11/16/83; Geometry: Modified Parabola; Focal Length $=6.00$; $\mathrm{f} / \mathrm{D}$ Ratio $=0.500$; 非 Rays $=3565$; 非 Circles $=100$; \# Points/Circle $=36$;

Plane Cut Z; Value: 6.030; Sigma $=14.00$;

Source Points: $X=0, Y=0, Z=900$ )

Normalized Aperture

Radius, mrad

\section{Intercept}

Factor

\subsection{7}

0.164

0.314

0.484

0.643

0.753

0.840

0.908

0.949

0.965

0.979

0.987

0.993

0.998

0.999

0.999

0.999

0.999

1.000
Points

Out

\section{3}

2982

2446

1839

1271

880

569

328

180

125

76

45

24

7

4

3

3

1

40.0 
Table 4-2b. Experimental Data for Figure 4-2

(Four Panel Experimental Data Source: Point, Visible Source Distance: $900 \mathrm{~m}$; Image Plane: $6.03 \mathrm{~m}$ )

\begin{tabular}{lrcr}
\multicolumn{2}{c}{$\begin{array}{c}\text { Aperture Radius } \\
\text { in. }\end{array}$} & Normalized Aperture \\
Radius, mrad & $\begin{array}{c}\text { Intercept Factor, } \\
\text { Measured }\end{array}$ \\
\hline 1.000 & 25.4 & 4.23 & 0.168 \\
1.500 & 38.1 & 6.35 & 0.350 \\
2.000 & 50.8 & 8.47 & 0.527 \\
2.500 & 63.5 & 10.58 & 0.695 \\
3.000 & 76.2 & 12.70 & 0.812 \\
3.500 & 88.9 & 14.82 & 0.881 \\
4.000 & 101.6 & 16.93 & 0.925 \\
4.500 & 114.3 & 19.05 & 0.946 \\
5.000 & 127.0 & 21.17 & 0.965 \\
5.500 & 139.7 & 23.28 & 0.977 \\
6.000 & 152.4 & 25.40 & 0.986 \\
6.500 & 165.1 & 27.52 & 0.990 \\
7.000 & 177.8 & 29.63 & 0.994 \\
7.500 & 190.5 & 31.75 & 0.997 \\
8.000 & 203.2 & 33.87 & 0.999 \\
& & & \\
\hline
\end{tabular}


Table 4-3. Model Computed Solar Source Projections for Figure 4-3

(PDC-1 Solar Source Projection Data Summary; Mode1 Parameters: Foca1 Length $=6.00 \mathrm{~m}$, Diameter $=12.00 \mathrm{~m}$, Source Distance $=1.5 \times 10^{11} \mathrm{~m}$, Image

Plane $=6.00 \mathrm{~m} ;\left(\mathrm{I} / \mathrm{I}_{0}\right)_{1}$ - Uniform Solar Disk, Radius $=5.0 \mathrm{mrad}$ and Surface Sigma $=16.25 \mathrm{mrad} ;\left(I / I_{O}\right)_{2}$ - Point Source (on axis) and Surface Sigma $=16.25 \mathrm{mrad} ;\left(I / I_{O}\right)_{3}-$ Uniform Solar Disk and Surface Sigma $=0.00 \mathrm{mrad}$ )

\begin{tabular}{|c|c|c|c|}
\hline $\begin{array}{l}\text { Normalized Aperture } \\
\text { Radius, mrad }\end{array}$ & $\begin{array}{l}\text { Intercept Factor } \\
\quad \text { (Curve 1) }\end{array}$ & $\begin{array}{c}\text { Intercept Factor } \\
\text { (Curve 2) }\end{array}$ & $\begin{array}{l}\text { Intercept Factor } \\
\quad \text { (Curve } 3 \text { ) }\end{array}$ \\
\hline 1.0 & - & - & 0.099 \\
\hline 2.0 & 0.027 & 0.036 & 0.262 \\
\hline 3.0 & - & - & 0.419 \\
\hline 4.0 & 0.097 & 0.124 & 0.578 \\
\hline 5.0 & - & - & 0.711 \\
\hline 6.0 & 0.221 & 0.247 & 0.873 \\
\hline 7.0 & - & - & 0.943 \\
\hline 8.0 & 0.353 & 0.383 & 0.977 \\
\hline 9.0 & - & - & 0.992 \\
\hline 10.0 & 0.479 & 0.515 & 0.999 \\
\hline 12.0 & 0.600 & 0.638 & - \\
\hline 14.0 & 0.700 & 0.734 & - \\
\hline 16.0 & 0.785 & 0.817 & - \\
\hline 18.0 & 0.852 & 0.873 & - \\
\hline 20.0 & 0.900 & 0.914 & - \\
\hline 22.0 & 0.935 & 0.949 & - \\
\hline 24.0 & 0.960 & 0.969 & - \\
\hline 26.0 & 0.974 & 0.983 & - \\
\hline 28.0 & 0.984 & 0.990 & - \\
\hline 30.0 & 0.991 & 0.996 & - \\
\hline 32.0 & 0.995 & 0.998 & - \\
\hline 34.0 & 0.998 & 0.999 & - \\
\hline 36.0 & 0.999 & 0.999 & - \\
\hline 38.0 & 0.999 & 0.999 & - \\
\hline 40.0 & 0.999 & 1.000 & - \\
\hline 42.0 & 0.999 & - & - \\
\hline 44.0 & 1.000 & - & - \\
\hline
\end{tabular}




